\title{
Un mariage forcé ? L'audiovisuel et les bibliothèques publiques
} An uneasy relationship? Audiovisual resources in public libraries

\section{¿Casamiento a la fuerza? Los recursos audiovisuales y las bibliotecas públicas}

\section{Silvie Delorme}

Volume 27, numéro 4, décembre 1981

URI : https://id.erudit.org/iderudit/1053794ar

DOI : https://doi.org/10.7202/1053794ar

Aller au sommaire du numéro

Éditeur(s)

Association pour l'avancement des sciences et des techniques de la documentation (ASTED)

ISSN

0315-2340 (imprimé)

2291-8949 (numérique)

Découvrir la revue

Citer cet article

Delorme, S. (1981). Un mariage forcé ? L’audiovisuel et les bibliothèques publiques. Documentation et bibliothèques, 27(4), 137-140.

https://doi.org/10.7202/1053794ar
Résumé de l'article

Nos bibliothèques publiques font peu de place à l'audiovisuel. L'auteur tente de démontrer que cette lacune est due à l'imprécision des buts de la bibliothèque publique et à la propension des bibliothécaires à favoriser le livre. Il précise ensuite ce que devrait être le rôle de la bibliothèque publique dans la société d'aujourd'hui et comment s'y rattache l'audiovisuel.
Tous droits réservés ( Association pour l'avancement des sciences et des techniques de la documentation (ASTED), 1981
Ce document est protégé par la loi sur le droit d'auteur. L'utilisation des services d'Érudit (y compris la reproduction) est assujettie à sa politique d'utilisation que vous pouvez consulter en ligne.

https://apropos.erudit.org/fr/usagers/politique-dutilisation/ 


\title{
Un mariage forcé? L'audiovisuel et les bibliothèques publiques
}

\author{
Silvie Delorme* \\ Bibliothèque municipale \\ Boucherville.
}

Nos bibliothèques publiques font peu de place à l'audiovisuel. L'auteur tente de démontrer que cette lacune est due à l'imprécision des buts de la bibliothèque publique et à la propension des bibliothécaires à favoriser le livre. II précise ensuite ce que devrait être le rôle de la bibliothèque publique dans la société d'aujourd'hui et comment s'y rattache l'audiovisuel.

\section{An uneasy relatlonship? Audlovisual resources in public Ilbrarles}

Little room is granted to audiovisual materials in our public libraries. The author tends to impute this lack of interest to the fact that the goals of the public library are yet to be clearly defined and that librarians favor books. She brings a new light on the understanding of the role of public libraries in today's society and the role that should be played by audiovisual media.

¿Casamlento a la fuerza? Los recursos audlovisuales y las blbliotecas públicas.

Nuestras bibliotecas públicas apenas ceden el paso a los recursos audiovisuales. El autor trata de demostrar que esta laguna se debe a la imprecisión de los objetivos de la biblioteca pública y a la propensión de los bibliotecarios a preferir el libro. Aclara, a continuación, tanto el papel que deberia desempeñar la biblioteca pública en la sociedad actual, como la forma en que se podrian integrar en ella los recursos audiovisuales.

Les Normes pour les bibliothèques publiques publiées récemment par le Service des bibliothèques publiques du Québec font très peu état de la part du budget à consacrer à l'audiovisuel. Celui-ci n'y est considéré que comme complément à la collection principale (lire ici livres) :

"La documentation imprimée devrait être complétée par des documents audiovisuels, en fonction des besoins locaux et dans la mesure où leur achat convient aux objectifs de la bibliothèque, mais jamais au détriment de la collection de base, et seulement lorsque le personnel et le budget sont suffisants"1

Qu'un organisme comme le Service des bibliothèques publiques, qui devrait donner le ton, stimuler les bibliothèques publiques et se placer à l'avant-garde, semble si peu persuadé de l'importance de l'audiovisuel dans les bibliothèques municipales peut étonner à bon droit. Les bibliothèques publiques du Québec doivent être conscientes de cette vision réduite des bibliothèques publiques que projette le Service et y remédier en développant un service audiovisuel adéquat.

\section{Buts et objectifs des bibliothèques publiques}

Depuis nombre d'années, les bibliothèques municipales cherchent leur voie et le but qu'elles poursuivent a changé à plusieurs reprises. Un ouvrage de Davies dresse

\footnotetext{
* L'auteur est responsable de l'animation à la bibliothèque municipale de Boucherville.

1. Ministère des Affaires culturelles du Québec, Service des bibliothèques publiques, Normes pour les bibliothèques municipales, Québec, 1981, p. 38.
} 
un bref historique de l'évolution de leur orientation².

Au début du XIXe siècle, les bibliothécaires ont commencé à s'intéresser à la notion de bibliothèque en tant que centre culturel. Cependant, même à cette époque, les institutions municipales entraient en conflit et en compétition avec d'autres organismes impliqués au plan culturel (musées, théâtres, centres culturels, sociétés musicales, groupes promouvant la formation des adultes...). Cette vision de leur rôle n'en permettait pas moins aux bibliothèques d'organiser des activités diverses dans le but d'augmenter leur niveau d'utilisation et de fréquentation, même si la culture devait passer par le livre. Les bibliothécaires de l'époque espéraient ainsi que ces nouveaux usagers deviennent des lecteurs. L'expérience a démontré la fausseté de cette philosophie.

Par la suite, la bibliothèque devint un centre social ayant pour but plus ou moins avoué l'intégration des minorités. Cette conception de la bibliothèque ne s'axait également que sur le livre:

«The intent of the modern library worker is to induce the poor to lead better lives, and the underlying belief is that the cold, the hungry and the penniless will lead better lives if they will only read. 3

Jussim souligne la même tendance en s'interrogeant sur les conséquences d'une telle prise de position:

«Excessive emphasis on print literacy, to the almost total exclusion of training and enlargment of other modes of apprehension, may result in deep frustration and a serious sense of alienation from the "real world ». 4

Ainsi, les bibliothèques entraient à nouveau en conflit avec certains organismes existants: les centres de services sociaux.

La bibliothèque publique n'aurait-elle donc pas de fonction propre et n'aurait-elle pour vocation que d'être constamment en concurrence avec d'autres organismes?

Quoiqu'on en dise ou pense, les buts et les objectifs de la bibliothèque publique n'étaient et ne sont toujours pas axés sur un concept, une orientation générale, une ligne directe, mais bien plutôt sur un support: le livre. Peu importe l'orientation donnée à la bibliothèque, elle ne vit et n'existe que par lui. Qu'elle ait une vocation culturelle ou sociale, le livre constitue sa raison d'être.

Cette conception est due aussi en partie à la vision qu'a le bibliothécaire de son rôle. Ce dernier n'a pas saisi l'importance de sa fonction dans la société. Il est demeuré attaché à un support, à un contenant plutôt que de s'arrêter à l'impact et à l'utilité du contenu du document. Sharr illustre bien cette constatation: "Books are to librarianship what bottles are to pharmacy: mere containers »5. Cet intérêt pour le contenu lui aurait permis de passer à l'audiovisuel sans problème puisqu'il aurait alors compris les analogies entre les divers média plutôt que de s'arrêter à leurs différences.

Les documents audiovisuels, avec des caractéristiques différentes, ont essentiellement les mêmes buts que l'imprimé : la communication et la diffusion des idées. Cet objectif correspond à la fonction du bibliothécaire contemporain.

\section{L'audiovisuel et les buts et objectifs des bibliothèques publiques}

La question de l'audiovisuel dans les bibliothèques publiques se pose avec acuité. Comment défendre la nécessité de l'audiovisuel dans une bibliothèque si cette dernière n'existe, en pratique, que par et pour le livre?

Bien sûr, les bibliothèques municipales poursuivent des buts: l'éducation, la culture, le divertissement et l'information d'une communauté donnée. Cependant, à y regarder de plus près, ces objectifs sont subordonnés au livre et non l'inverse, comme il se devrait. Ce qui permettrait d'intégrer tous les supports existants à la bibliothèque.

Ne faudrait-il pas avoir une vision globale, une perspective d'ensemble, une ligne de conduite qui se focaliseraient sur un but en particulier, autour duquel

2. D.W. Davies, Public Libraries as Culture and Social Centers : The Origin of the Concept, Metuchen, Scarecrow, 1974, passim.

3. Ibid., passim.

4. E. Jussim, "Confronting media biases : the social dimensions of media theory ", School Libraries, vol. 21, no. 4 (Summer 1972), 16.

5. F.A. Sharr, "The public library: Dodo or Phoenix. New directions in public library policy ", Libri, vol. 24, no. 2 (1974), 90. 
s'organiseraient, s'imbriqueraient toutes les activités de la bibliothèque?

Vus sous cet angle, il est facile d'expliquer les problèmes que rencontrent les bibliothécaires municipaux lorsqu'ils veulent défendre auprès des conseils municipaux les budgets de l'animation ou de l'audiovisuel, puisque eux-mêmes rattachent difficilement ces domaines au livre ou le font de façon peu convaincante.

L'utilisation des notions de marketing éviterait tous ces ennuis. Le marketing consiste à définir un produit selon les besoins des usagers par le biais des études de marché. Les bibliothèques municipales ne circonscrivent pas leur produit d'après les besoins de la communauté. Elles partent au contraire de leur produit: le livre (déterminé il y a des siècles), essaient de connaître les besoins des citoyens en livres et de leur vendre leurs services. Ce que constate Croneberger ${ }^{6}$ :

"We are marketing the wrong product. We are still selling books while the public needs and wants information in all its forms. "

en faisant, pour illustrer son propos, la comparaison suivante: un fabricant de charettes qui tenterait de vendre son produit aujourd'hui serait voué à la faillite à moins qu'il ne soit adapté aux besoins du transport moderne, et qu'il ait transformé sa charette en automobile.

Les bibliothèques municipales sont confrontées au même problème. II ne s'agit plus de vendre leur produit, le livre, mais de répondre aux besoins de la communauté : l'information, et ainsi d'adapter les services offerts aux diverses demandes du public en donnant accès à plusieurs supports susceptibles de combler ce besoin d'information.

"Marketing demands that business define its goals as the satisfaction of customer needs. It does not ask, "What do we want to sell ? » It asks "What does the customer want to buy? " It does not say "This is what our product or service does." It says "These are the satisfactions the customer looks for, values and needs. $" 7$

Sharr nous propose aussi cette vision d'une bibliothèque adaptée à notreépoque, consciente des besoins des individus et de la communauté, visant à satisfaire les besoins en information des citoyens:

"The professional role of the librarian is, typically, to introduce people to relevant information... His preoccupation should be with people; the contents of his library and its organisation are but the means at his disposal to achieve his purpose. $\gg 8$

Contrairement à ce que l'on pourrait penser, rattacher la bibliothèque publique à l'information ne la réduit pas à un seul champ d'activité. L'information peut avoir plusieurs buts. Elle peut être, de par sa nature et son utilisation, éducative, culturelle et peut même contribuer au divertissement. Sharr entend également information dans un sens très large incluant la fiction et le divertissement. ${ }^{9}$

L'information peut prendre différentes formes: écrite (livres, périodiques, journaux), visuelle (diaporamas, films fixes, maquettes...), audio (disques, cassettes), audiovisuelle (films, vidéo...), personnelle (conférences, kiosques d'information, information communautaire, services de référence...). Ainsi, la bibliothèque devient un organisme d'information au même titre que la radio et la télévision, à cette différence près que l'individu va chercher lui-même ce qui l'intéresse.

L'audiovisuel est automatiquement englobé dans cette vision et se justifie ainsi très facilement. Comme le souligne Goldstein à propos du vidéo :

"Video technology can help (to) reach people who are not print-oriented and

(to) disseminate information in a format with which many are comfortable (T.V.) $) 10$

Tous ne sont pas à l'aise au même degré avec l'imprimé. L'audiovisuel permet d'informer les citoyens par le biais du média qui leur est le plus familier. Harrisson appuie ce point de vue lorsqu'elle fait le raisonnement suivant: si le matériel

6. R. Croneberger Jr., "Effective public library", Catholic Library World, vol. 48 (November 1976) 168.

7. Peter F. Ducker, “Management: tasks, responsibilities, practices", cité dans Croneberger, ibid., 169.

8. F.A. Sharr, "The public library...", 90.

9. Ibid.

10. Seth Goldstein, Video in Libraries. A Status Report. 1977-1978, White Plains, N.Y., Knowledge Industry Publications, 1977, p. 7 
audiovisuel contient de l'information et que la bibliothèque est directement intéressée par l'information, alors la bibliothèque est inévitablement intéressée à l'audiovisuel. De plus, elle souligne que l'imprimé et l'audiovisuel sont des partenaires et non des rivaux comme nous le concevions il y a quelques années. ${ }^{11}$

Veaner abonde dans le même sens:

"Human beings require a spectrum of media to serve their graphic and nongraphic needs. The one medium that serves every purpose doesn't exist and probably never will. »12

Flanagan rejoint enfin ses collègues lorsqu'il précise que les bibliothèques n'offrent pas toutes les ressources disponibles souhaitables: "After all books are only a small fraction of the devices men have for communicating. »13

\section{Le matériel audiovisuel}

Dans cette nouvelle perspective, l'audiovisuel prend une place importante dans la bibliothèque et doit faire l'objet d'une bonne planification.

Selon des auteurs connus ${ }^{14}$, il importe avant d'instaurer un service audiovisuel dans une bibliothèque publique d'établir la politique de la bibliothèque en ce domaine, de préciser les besoins de la clientèle, d'élaborer un service audiovisuel en fonction de ces besoins et de ces intérêts et d'identifier les activités nécessaires à l'implantation du service.

Pour bien connaître la communauté à desservir, il faut franchir plusieurs étapes : fixer les buts et les responsabilités de la bibliothèque face à la communauté ; déterminer à quels groupes appartiennent les usagers et les non-usagers ; étudier les besoins en information et les intérêts de chacun des groupes d'usagers identifiés en se demandant si l'audiovisuel serait approprié pour ces groupes et pourquoi ; refaire le même processus pour connaître les besoins et les intérêts des non-usagers, déterminer selon les besoins des différents groupes d'usagers le type de document audiovisuel le plus pertinent, refaire ce même exercice pour les non-usagers.

Cette façon de procéder permet d'identifier le support audiovisuel le mieux adapté aux besoins des usagers et des non-usagers et de planifier le développement de la collection en conséquence.

II est évident que certains documents audiovisuels, les films surtout $(16 \mathrm{~mm}$ et super 8 ), sont très coûteux et que toutes les bibliothèques ne peuvent se permettre un tel luxe. Cependant, avec l'ère du vidéo, il sera possible d'offrir des films à des coûts plus raisonnables. Le prix des vidéo-cassettes, selon la durée, varie entre $\$ 50.00$ et $\$ 150.00-\$ 200.00$ et celui des vidéo-disques variera entre $\$ 15.00$ et $\$ 20.00$ lorsqu'ils pourront être produits en grande quantité.

La télévision sur demande à la bibliothèque constitue également une approche intéressante, peut-être plus lointaine mais non moins possible.

Outre les documents audiovisuels traditionnels (films, disques, cassettes, diaporamas...), il existe, avec un peu d'imagination, d'autres documents audiovisuels utilisables en bibliothèque. Les maquettes et les objets en trois dimensions constituent des documents visuels peu utilisés mais qui auraient avantage à l'être. Un moteur d'automobile en modèle réduit, par exemple, serait sûrement très utile au mécanicien amateur qui désire se familiariser avec la mécanique. Que dire des maquettes de maisons traditionnelles du Québec pour l'amateur d'architecture, des bâteaux, des avions, des trains et des fusées pour les jeunes... Les utilisations possibles de ces objets sont nombreuses, intéressantes et variées.

Dans notre société, pour survivre, il faut répondre aux besoins du public. La bibliothèque n'échappe pas à cette contrainte et doit répondre aux besoins en information des citoyens. Non seulement en théorie, c'est-à-dire en acceptant les principes tout en ne continuant de vivre que pour le livre, mais en offrant tous les supports susceptibles de communiquer cette information dont le citoyen a besoin. Les supports audiovisuels sont de ceux-là.

11. Helen P. Harrisson, "Progress in documentation. Non-books materials : a decade of development", Journal of Documentation, vol. 35, no. 3 (Sept. 1979), 308.

12. A.B. Veaner, "Hurray for meshed mediums", Library Journal, vol. 103, no. 14 (August 1978), 1449.

13. L.N. Flanagan, «Disestablishing the school and the public library: the ideas of Ivan Illich applied to libraries ", California Librarian, vol. 32 (July 1974), 60.

14. S. Mcintyre-Johnson, L. Schexnaydre, M.S. Woods, Planning Audiovisual Services in Public Libraries, Austin, Texas State Library, Department of Library Development, 1975. 276 p. 